\title{
Stronger pharmacological cortisol suppression and anticipatory cortisol stress response in transient global amnesia
}

\section{Martin Griebe ${ }^{1 *}{ }^{\dagger}$, Frauke Nees ${ }^{2 \dagger}$, Benjamin Gerber ${ }^{1}$, Anne Ebert ${ }^{1}$, Herta Flor ${ }^{2}$, Oliver T. Wolf ${ }^{3}$, Achim Gass ${ }^{1}$, Michael G. Hennerici ${ }^{1}$ and Kristina Szabo'}

${ }^{1}$ Department of Neurology, UniversitätsMedizin Mannheim, Heidelberg University, Mannheim, Germany

${ }^{2}$ Department of Cognitive and Clinical Neuroscience, Central Institute of Mental Health, Heidelberg University, Mannheim, Germany

${ }^{3}$ Department of Cognitive Psychology, Institute of Cognitive Neuroscience, Ruhr-University Bochum, Bochum, Germany

\section{Edited by:}

Edo Ronald De Kloet, Leiden University, Netherlands

Reviewed by:

Tony W. Buchanan, Saint Louis University, USA

Jianhui Wu, Chinese Academy of Sciences, China

\section{*Correspondence:}

Martin Griebe, Department of Neurology, UniversitätsMedizin Mannheim, Theodor-Kutzer-Ufer 1-3, Mannheim 68135, Germany e-mail: griebe@neuro.ma.

uni-heidelberg.de

${ }^{+}$Martin Griebe and Frauke Nees have contributed equally to this work.

\begin{abstract}
Transient global amnesia (TGA) is a disorder characterized by a sudden attack of severe anterograde memory disturbance that is frequently preceded by emotional or physical stress and resolves within $24 \mathrm{~h}$. By using MRI following the acute episode in TGA patients, small lesions in the hippocampus have been observed. Hence, it has been hypothesized that the disorder is caused by a stress-related transient inhibition of memory formation in the hippocampus. To study the factors that may link stress and TGA, we measured the cortisol day-profile, the dexamethasone feedback inhibition and the effect of experimental exposure to stress on cortisol levels (using the socially evaluated cold pressor test and a control procedure) in 20 patients with a recent history of TGA and in 20 healthy controls. We used self-report scales of depression, anxiety and stress, and a detailed neuropsychological assessment to characterize our collective. We did not observe differences in mean cortisol levels in the cortisol day-profile between the two groups. After administration of low-dose dexamethasone, TGA patients showed significantly stronger cortisol suppression in the daytime profile compared to the control group $(p=0.027)$. The mean salivary cortisol level was significantly higher in the TGA group prior to and after the experimental stress exposure ( $p=0.008$ and 0.010 respectively), as well as prior to and after the control condition ( $p=0.022$ and 0.024 , respectively). The TGA group had higher scores of depressive symptomatology $(p=0.021)$ and anxiety $(p=0.007)$, but the groups did not differ in the neuropsychological assessment. Our findings of a stronger pharmacological suppression and higher cortisol levels in anticipation of experimental stress in participants with a previous TGA indicate a hypersensitivity of the HPA axis. This suggests that an individual stress sensitivity might play a role in the pathophysiology of TGA.
\end{abstract}

Keywords: transient global amnesia, cortisol, stress, hippocampus, memory

\section{INTRODUCTION}

Transient global amnesia (TGA) is characterized by a sudden attack of severe disturbance of anterograde episodic long-term memory disrupting the learning of novel episodic information (Mazzucchi and Parma, 1990; Jager et al., 2009) that resolves within $24 \mathrm{~h}$ (Hodges and Warlow, 1990). Several groups have suggested that the pathophysiological mechanisms leading to TGA may be similar to those of cerebral ischemia, epilepsy, or migraine (Frederiks, 1993). However, there is no definitive evidence supporting any of these mechanisms. More recently, a disturbance of venous hemodynamics has been hypothesized, but again without scientific data for such an underlying mechanism (Baracchini et al., 2012).

Abbreviations: AUCg, area under the curve with respect to ground; $\mathrm{AUCi}$, area under the curve with respect to increase; CES-D, center for epidemiological studies depression scale; HPA axis, hypothalamic-pituitary-adrenal axis; SECPT, socially evaluated cold pressor test; STAI, state-trait anxiety inventory; TGA, transient global amnesia; TICS, trier inventory for chronic stress.
Due to the clinical characteristics of the cognitive impairment during TGA, a transient dysfunction of the medial temporal lobes, especially of the hippocampus, has been postulated (Bartsch and Deuschl, 2010). In up to $80 \%$ of patients, diffusion-weighted MRI has been reported to show small lesions in the hippocampus 24$48 \mathrm{~h}$ after symptom onset (Gass et al., 1999; Sedlaczek et al., 2004; Bartsch et al., 2007). While this finding actually links the disorder to the CA-1 subfield of the hippocampus anatomically, the exact etiology of these lesions remains uncertain. Interestingly, physically or emotionally stressful episodes have been reported as precipitating events in up to $89 \%$ of TGA cases (Quinette et al., 2006). These observations have led to the implication that stress might play a role in the pathophysiology of TGA. More precisely, it has been hypothesized that TGA might be caused by a stress-related transient inhibition of memory formation in the hippocampus by means of a selective vulnerability of CA-1 neurons to metabolic stress (Bartsch and Deuschl, 2010). A recent study suggests that an elevated anxiety level may increase the susceptibility to 
psychological stress, which may facilitate the pathophysiological cascade in TGA (Dohring et al., 2014), however, the neuroendocrine response via the hypothalamic-pituitary-adrenal (HPA) axis has not been characterized.

To study the factors that may link psychological or physical stress to TGA on a neuroendocrine level, we measured the cortisol day-profile, dexamethasone feedback inhibition, and the effect of experimental exposure to stress on cortisol levels in 20 patients with a recent history of TGA and in 20 healthy controls. As experimental stress procedure, we used the socially evaluated cold pressor test (SECPT) during which subjects immerse their hand in ice water and are observed and videotaped by an unfamiliar experimenter. We used self-report scales of depression, anxiety, and stress and a detailed neuropsychological assessment to characterize our collective. We hypothesized that TGA patients exhibit a stronger HPA axis reactivity compared to the control subjects.

\section{MATERIALS AND METHODS PARTICIPANTS}

From our prospectively collected TGA database with 208 cases since 2001 fulfilling the established criteria (Hodges and Warlow, 1990), we contacted those 41 who had had a TGA in the years 2010 and 2011. Out of 26 patients willing to participate in the study, we included 20 right-handed TGA patients. Six patients were excluded due to meeting the following pre-specified exclusion criteria: left-handedness $(n=3)$, co-medication (corticosteroids, $n=2$ ), and comorbidity interfering with the conduct of the trial (tremor, $n=1$ ). We recruited 20 controls matched for age, sex, and education (see Table 1). The study received ethics approval, and all participants gave written informed consent. The study examinations were performed on three days: day 1 (in the morning) - neuropsychological evaluation, instruction for cortisol sample collection; day 2 and day 3 one-week apart at the same

Table 1 | Characteristics of the study population

\begin{tabular}{lccc}
\hline & TGA & Controls & $\boldsymbol{p}$ value \\
\hline Number & 20 & 20 & - \\
Age, years; mean (SD) & $66.50( \pm 7.7)$ & $66.55( \pm 7.0)$ & 0.940 \\
Sex, male; number & 8 & 8 & 1.000 \\
Formal education, years; & $12.5(8-19)$ & $13(8-17)$ & 0.557 \\
median (range) & & & \\
Participants with mental & 6 & 1 & $\mathbf{0 . 0 2 3}$ \\
disordera; number & & & \\
MMSE, sum; median (range) & $30(28-30)$ & $29.5(28-30)$ & 0.869 \\
Time since TGA, months; & $10.55(11.15)$ & NA & - \\
mean (SD) & & & \\
Participants with DWI lesion; & 16 & & \\
number & & & \\
$\quad$ Left hippocampus & 4 & & \\
Right hippocampus & 2 & & \\
Bilateral hippocampi & 10 & &
\end{tabular}

DWI, diffusion-weighted MRI; MMSE, mini mental state examination; SD, standard deviation.

Bold font indicates $p<0.05$.

a Previously diagnosed depression or anxiety disorder. time of day (between 1 and 4 p.m.) - stress exposure or control condition in a randomized order.

\section{HPA AXIS ACTIVITY}

Saliva samples were collected into Salivette tubes (Sarstedt, Nümbrecht, Germany), immediately after as well as $15,30,45$, and $60 \mathrm{~min}$ after awakening (cortisol awakening response; SchmidtReinwald et al., 1999) and at 11 a.m., 1 p.m., 3 p.m., 6 p.m., 8 p.m., 10 p.m., and 11 p.m. (daytime profile). Salivary cortisol values were also sampled in an identical fashion on a second day following the administration of $0.5 \mathrm{mg}$ dexamethasone at 11 p.m. (lowdose dexamethasone suppression test/dexamethasone challenge). Participants were instructed to refrain from smoking (all participants were non-smokers), drinking, or eating $10 \mathrm{~min}$ and brushing one's teeth 5 min before each sampling. Participants completed a diary during the sampling period containing information on sleep duration, food intake, and daytime activities. Self-reports as well as an electronic monitoring device (MEMS Track Cap, Aardex, Switzerland) were employed to verify sample times. Cortisol data of two participants on day 1 (one TGA, one control) and three participants on day 2 (two TGA, one control) were excluded due to incongruence between self-report and electronic data. Saliva samples collected into Salivette tubes (Sarstedt, Nümbrecht, Germany) were kept at $-20^{\circ} \mathrm{C}$ until analysis. Free cortisol levels in saliva were measured using a commercially available immunoassay (IBL, Hamburg, Germany) at the Institute of Biopsychology, Technische Universitaet Dresden, Dresden, Germany. Intra- and interassay variances were below $10 \%$.

\section{STRESS PROTOCOL}

All participants were exposed to the SECPT (Schwabe et al., 2008). They were advised to immerse their right hand including the wrist in ice water $\left(0-4^{\circ} \mathrm{C}\right)$ for $3 \mathrm{~min}$ or until they could no longer tolerate it. During the procedure, they were supervised by an unfamiliar person and informed that they were being videotaped for analysis of facial expression. All participants also underwent the control condition with warm water $\left(35-37^{\circ} \mathrm{C}\right)$ during which they were not filmed. The order of the two procedures was randomized. Immediately after the SECPT and the control condition, participants were asked to rate how stressful, painful, and unpleasant the experiment had been ( 0 "not at all" to 100 "very"). Blood pressure (auscultatory method with stethoscope and aneroid sphygmomanometer), heart rate (manual palpation of radial artery), and salivary cortisol values were collected before, after 3 and after $15 \mathrm{~min}$.

\section{SELF-REPORT SCALES OF DEPRESSION, ANXIETY, AND STRESS}

The German version of the CES-D (Hautzinger and Bailer, 1993) was used to measure depressive symptomatology. State and trait of anxiety were measured using the German version of the STAI (Laux et al., 1981) consisting of 40 questions on a self-report basis. The Trier inventory for chronic stress (TICS; Schulz and Schlotz, 1999) was employed to assess the level of chronic psychosocial stress arising from environmental and internal demands in several domains in a defined time frame.

\section{NEUROPSYCHOLOGICAL ASSESSMENT}

All participants underwent a standardized neuropsychological assessment of the following domains: global cognitive function 
[Mini Mental State Examination (Folstein et al., 1975)], semantic memory [recognition vocabulary from the Achievement Measure System (Horn, 1983)], semantic word fluency [Regensburg Vocabulary Test (Aschenbrenner et al., 2001)], reaction time [alertness assessment from the Test for Attentional Performance (Zimmermann and Fimm, 2012)], executive functions [lexical fluency from the Regensburg Vocabulary Test (Aschenbrenner et al., 2001)]; selective attention in Go-Nogo of the Achievement Measure System (Zimmermann and Fimm, 2012), visuo-spatial functions [mental rotation and figure-ground perception of the Achievement Measure System (Horn, 1983)], verbal and non-verbal shortterm memory [digit and block span forwards of the Revised Wechsler Memory Scale (Härting et al., 2000)], verbal and non-verbal working memory [digit and block span backwards of the Revised Wechsler Memory Scale (Härting et al., 2000)], verbal episodic memory [Auditory Verbal Learning Test (Helmstaedter and Lux, 2001)], and visual episodic memory [route learning and retrieval of the Visual and Verbal Memory Test (Schellig and Schächtele, 2009); pattern recognition of the (CANTABeclipse, 2006)].

\section{STATISTICAL ANALYSIS}

Neuropsychological test results and self-ratings were analyzed by independent samples $t$-tests or Mann-Whitney- $U$-tests as appropriate. Group differences in the circadian profiles of cortisol concentrations between TGA patients and controls were calculated by one-way repeated measures ANOVA with 12 within-group levels. To compare cortisol levels for each sampling point, we used independent samples $t$-tests. Area under the curve with respect to ground (AUCg) and area under the curve with respect to increase (AUCi) were calculated for both circadian profiles of cortisol and compared between groups by independent samples $t$-tests (Pruessner et al., 2003). Group differences in cortisol, blood pressure, and heart rate responses to SECPT and the control procedure were calculated by one-way repeated measures ANOVA with three within-group levels. Thereafter, data for each of the three sampling points were compared by independent samples $t$-tests and alterations in the time course were calculated by dependent samples $t$-tests. To compare duration of SECPT between groups, we performed independent samples $t$-tests, to compare self-ratings of reactive stress and pain, Mann-Whitney- $U$-tests were performed. Pearson's correlation coefficient was calculated to test for a linear correlation between cortisol levels before the SECPT or the control procedure and the results of CES-D, STAI, and TICS, as well as between the stress reactivity (difference last cortisol level after SECPT or the control procedure and cortisol level before) and the results of CES-D, STAI, and TICS.

\section{RESULTS}

\section{STUDY POPULATION}

Table 1 summarizes the characteristics of the study population. The TGA and control group did not differ significantly with respect to age, sex, education, and MMSE score. Patients with a former TGA had a significantly ( $p=0.023$ ) higher incidence of a previously diagnosed mental disorder (TGA: depression, $n=5$, anxiety disorder, $n=1$; controls: depression, $n=1)$. Sixteen patients showed a hippocampal diffusion-weighted MRI lesion after the TGA episode (see Figure 1).

\section{CORTISOL STATUS}

We found no significant differences in mean cortisol levels during the awakening response or in the daytime cortisol profile between the two groups. After administration of low-dose dexamethasone, the TGA patients showed significantly stronger cortisol suppression in the daytime profile compared to the control group $(p=0.027)$. For details see Figure 2 and Table 2 .

\section{SOCIALLY EVALUATED COLD PRESSOR TEST}

None of the participants suffered any adverse effects (including headaches) after the stress exposure. The mean salivary cortisol level was significantly higher in participants with a history of TGA already prior to $(p=0.008)$ and immediately after the SECPT $(p=0.010)$ than in those without (see Table 2). After an additional $15 \mathrm{~min}$, salivary cortisol values were not different between the TGA group and those without TGA $(p=0.281)$. We found a significant increase of cortisol in the control group between the first and last measurements (before and $15 \mathrm{~min}$ after SECPT; $p=0.002$ ), but not in the TGA group $(p=0.171)$.

Interestingly, during the control condition, we made a similar observation: The mean salivary cortisol level was significantly higher in participants with a history of TGA already prior to $(p=0.022)$ and immediately after the $3 \mathrm{~min}$ warm water procedure $(p=0.024)$ than in the control group. After an additional $15 \mathrm{~min}$, salivary cortisol values were not significantly different between the groups $(p=0.134)$. During this experiment, the TGA group showed a decrease in cortisol levels from the first to the third measurement. However, this decrease was not statistically significant $(p=0.099)$.

Repeated measures analysis for interaction between groups missed significance $(p=0.082)$. Figure 3 shows the change across the three time points during both procedures for participants with a history of TGA and the controls. Eight participants (six TGA and two controls) did not immerse their hand for the full $3 \mathrm{~min}$ in ice water (TGA group mean duration $=146 \mathrm{~s}$, range: $28-180 \mathrm{~s}$; control group mean duration $=169 \mathrm{~s}$, range $50-180 ; p=0.065$ ). The TGA group perceived the SECPT as significantly more stressful $(p=0.036)$ but not as more painful $(p=0.130)$. There were no significant differences in increase of systolic or diastolic blood pressure or heart rate between the two groups during the SECPT.

\section{SELF-REPORT SCALES AND NEUROPSYCHOLOGICAL ASSESSMENT}

The TGA group had higher levels of depressive symptomatology and higher scores of anxiety compared with the control group as assessed by the CES-D $(p=0.021)$ and the STAI $(p=0.007)$, respectively (see Table 3 ). There was no significant difference between the two groups for chronic stress as measured by the TICS $(p=0.134)$.

There were no significant differences in the results of the neuropsychological assessment between the two groups in any of the used measures: semantic memory $(p=0.686)$, semantic word fluency $(p=0.929)$, reaction time $(p=0.326)$, executive functions (lexical fluency $p=0.884$; selective attention speed $p=0.146$; false positive reactions $p=0.057$ ), visuo-spatial functions (mental rotation $p=0.798$ and figure-ground perception $p=0.949$ ), verbal and non-verbal short-term memory (digit $p=0.774$ and block 

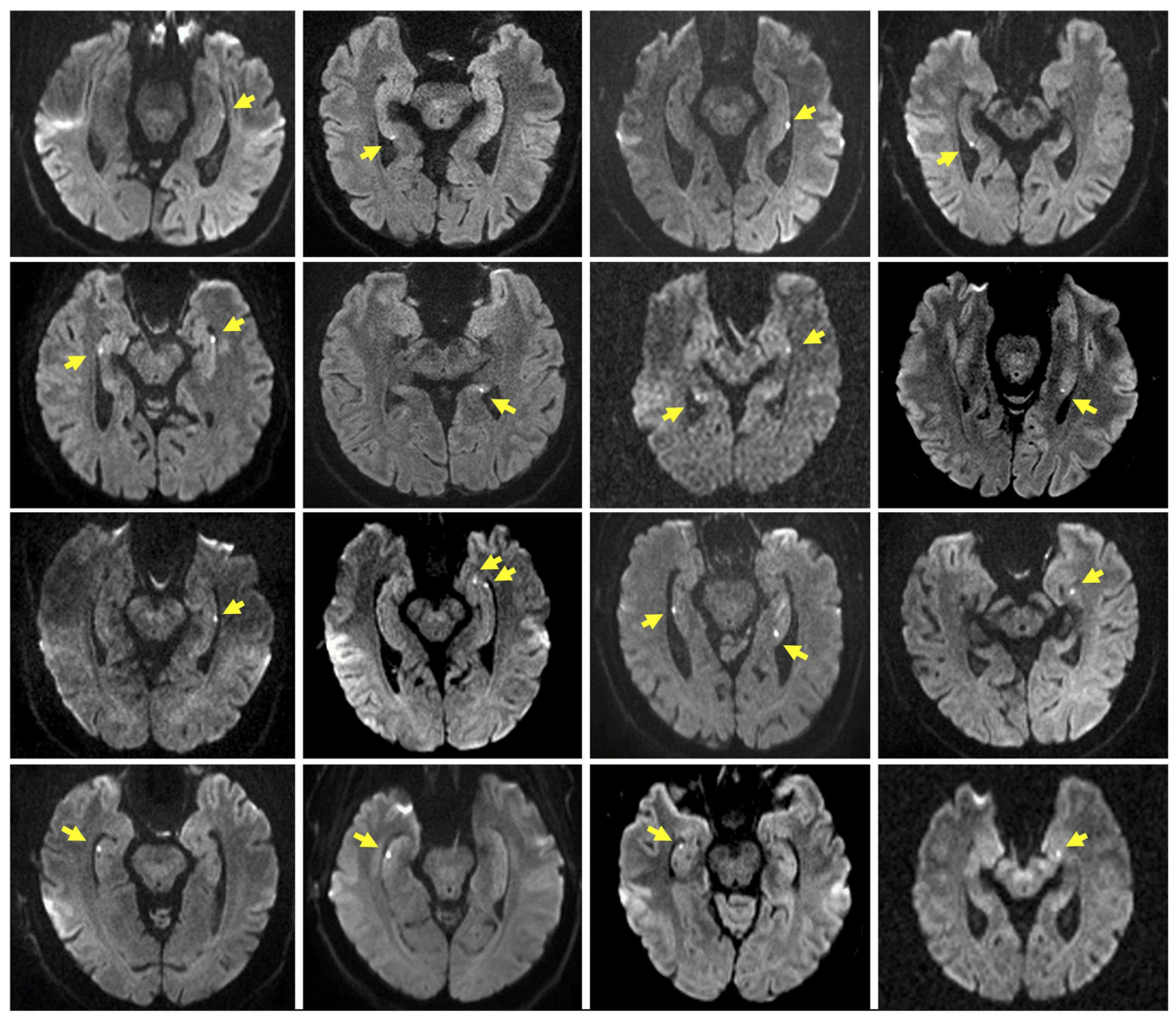

FIGURE 1 | Hippocampal MRI in TGA patients. Representative diffusion-weighted MR images after the acute episode of TGA in 16 of the 20 patients. Slice positioning parallel to the long axis of the hippocampus. Yellow arrows indicate the hippocampal lesions.

span forward $p=0.704)$, verbal and non-verbal working memory (digit $p=0.848$ and block span backward $p=0.842$ ), verbal episodic memory (learning, sum of trials $1-5, p=0.434$; retroactive interference trials 5 and $6, p=0.200$; late recall loss, trials $5-7, p=0.125$; recognition, hits - false positives $p=0.628)$, and visual episodic memory (route learning $p=0.836$ and retrieval $p=0.926$; immediate recognition $p=0.150$; delayed recognition $p=0.927)$.

\section{CORRELATIONS WITH CORTISOL RESPONSE}

We found no correlation between the first cortisol value before the SECPT or before the control procedure and the CES-D, STAI, or TICS scores in either group. Similarly, there was no correlation between the CES-D, STAI, or TICS score and the stress reactivity, defined as the difference between the last and the first cortisol measurement of the SECPT or the control procedure.

\section{DISCUSSION}

In this study, we aimed to investigate the possible role of stress in TGA. We observed a significantly stronger daytime suppression of cortisol secretion in response to low-dose dexamethasone and higher levels of cortisol in TGA patients before and after an experimental stress condition but also before and after the control procedure.

The possible role of certain personality traits - making individuals susceptible to stress - has been reported in TGA patients. Certain aspects of personality have been suggested to be more common in TGA: Inzitari et al. (1997) found phobic personality traits in $82 \%$ of 51 TGA patients. Also, depressive symptoms and comorbidities with mental disorder have been claimed to be more common in TGA (Neri et al., 1995; Pantoni et al., 2005). In their series of 142 patients, Quinette et al. (2006) reported a high frequency of psychological and emotional instability suggesting that TGA patients might be particularly vulnerable to psychological stress. Similarly, a recent study came to the conclusion that TGA patients might cope with stress less efficiently and less constructively than controls (Dohring et al., 2014). The authors report that patients with a stress-related precipitating event had a higher level of anxiety as measured by self-rating questionnaires in comparison to patients without such an event and controls. Our study confirms previous observations of higher levels of depressive symptomatology, higher scores of anxiety, as well as a higher prevalence of preexisting psychiatric comorbidities in TGA patients. Concerning the reversibility of memory deficits in TGA, many studies have shown 


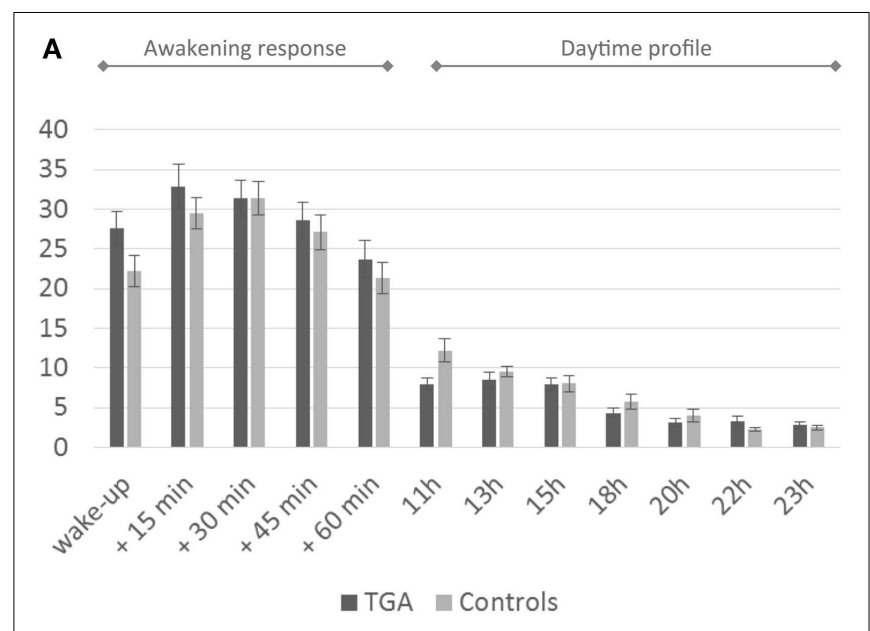

B Awakening response $\longrightarrow, \quad$ Daytime profile*

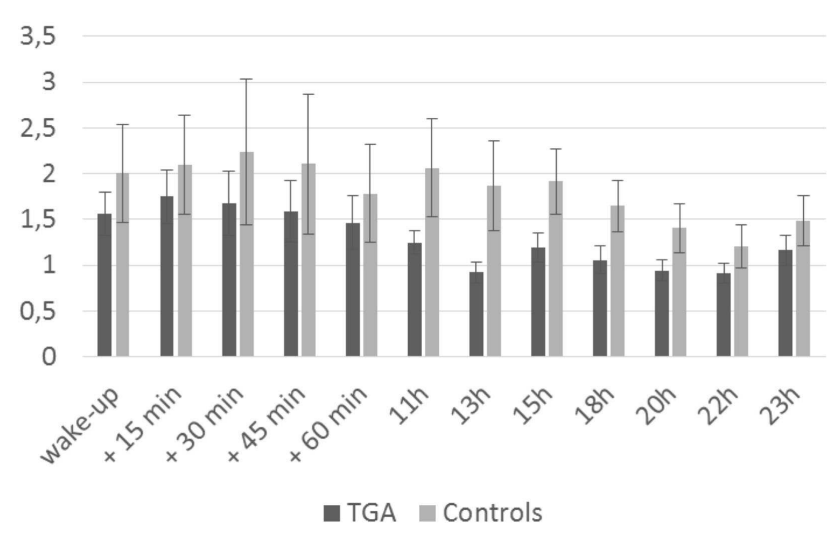

FIGURE 2 | Cortisol profiles. (A) Daytime profile of salivary cortisol levels. Twenty-four-hour cortisol profile in 20 participants with a history of TGA and 20 age and sex matched controls. Although the cortisol awakening response is higher in the TGA group, this is not significant $(p=0.140)$. (B) Salivary cortisol levels after dexamethasone challenge. Twenty-four-hour cortisol profile in 20 participants with a history of TGA and 20 age and sex matched controls following the administration of $0.5 \mathrm{mg}$ dexamethasone at 11 p.m. on the day before. TGA patients show a significantly stronger cortisol suppression in the daytime profile compared to the control group $(p=0.027)$. Data are presented as the mean (in $\mathrm{nmol} / \mathrm{l})$ at predefined sample times (awakening, $+15,+30,+45,+60$ min, thereafter daytime profile) across subjects. ${ }^{*}$ indicates significant group difference.

complete recovery of memory functioning several months after an episode of TGA (Kritchevsky et al., 1988; Kritchevsky and Squire, 1989; Faglioni et al., 1992; Quinette et al., 2003; Bartsch et al., 2006; Uttner et al., 2007). Similarly, we found no significant differences in the results of the neuropsychological assessment between groups.

Already in 1964, the presence of certain events occurring immediately before the attack - such as swimming in cold water, taking hot showers, pain, and sexual intercourse - was described (Fisher and Adams, 1964). As more recently analyzed, in up to $90 \%$ (32$89.1 \%$ ) of reported TGA cases, a precipitating event - mainly described as physical, emotional, or behavioral stress - has been reported (Quinette et al., 2006). In addition to those mentioned
Table 2 | Cortisol profiles

TGA Controls $p$ value

\section{Cortisol status}

AUCg; nmol/l h; mean (SEM)

$\begin{array}{llll}\text { Cortisol awakening response, } & 8.11( \pm 8.66) & 20.91( \pm 7.38) & 0.140\end{array}$

AUCi; nmol/l h; mean (SEM)

Cortisol daytime profile,

$68.03( \pm 4.34) \quad 78.02( \pm 6.27) \quad 0.105$

AUCg; nmol/l h; mean (SEM)

DST awakening response,

AUCg; nmol/l h; mean (SEM)

DST awakening response,

AUCi; nmol/l h; mean (SEM)

DST daytime profile, AUCg;

$\mathrm{nmol} / \mathrm{h}$; mean (SEM)

$\begin{array}{ccc}6.53( \pm 1.11) & 2.85( \pm 2.61) & 0.273 \\ 0.28( \pm 0.95) & 0.33( \pm 0.89) & 0.486 \\ 12.53( \pm 1.20) & 20.06( \pm 3.41) & \mathbf{0 . 0 2 7}\end{array}$

\section{Socially evaluated cold} pressor test SECPT, nmol/l; mean (SEM)

Salivary cortisol level after

SECPT, nmol/l; mean (SEM)

Salivary cortisol level after

$15 \mathrm{~min}, \mathrm{nmol} / \mathrm{l}$; mean (SEM)

$\begin{array}{crr}11.28( \pm 1.44) & 7.12( \pm 0.75) & \mathbf{0 . 0 0 8} \\ 11.81( \pm 1.34) & 7.92( \pm 0.86) & \mathbf{0 . 0 1 0} \\ 13.72( \pm 2.08) & 12.25( \pm 1.41) & 0.281\end{array}$

Salivary cortisol level before

AUCg, area under the curve with respect to ground; AUCi, area under the curve with respect to increase; DST, dexamethasone suppression test; SEM, standard error of the mean.

Bold font indicates $p<0.05$.

above, typical such events include stressful medical examinations, arguments, legal proceedings, funerals, exhausting physical work, and celebrations (Schmidtke and Ehmsen, 1998; Quinette et al., 2006; Griebe et al., 2015). In addition to events immediately before the attack, remote factors preceding TGA by days or weeks, for example, anxiety, exhaustion, and financial worries have also been described (Quinette et al., 2006). We used the SECPT, an experimental stress procedure that has been shown to lead to an HPA axis system activation (Schwabe et al., 2008). This stressor seems very appropriate, as immersion in cold water has not only been reported in case series of TGA leading to the term "amnesia by the seaside" (Martin, 1970), but has also been reported to lead to an amnestic episode in an experimental setting (Castellani et al., 1998). In our study, TGA patients exhibited elevated cortisol levels already before and immediately after both the SECPT and the control condition compared to the control subjects. Due to the physiologically slow HPA axis mediated cortisol response, the values obtained at these two timepoints cannot be regarded as the reactive cortisol response, but rather as an anticipatory cortisol response (Engert et al., 2013). Since the procedures were scheduled in a blinded and randomized order, we assume that TGA patients reacted to the upcoming and potentially stressful experiment. The anticipatory cortisol response can be interpreted as a manifestation of increased subjective stress sensitivity. This is in line with observations in posttraumatic stress disorder (Bremner et al., 2003), phobia (Alpers et al., 2003), and alexithymia (de Timary et al., 2008). The 

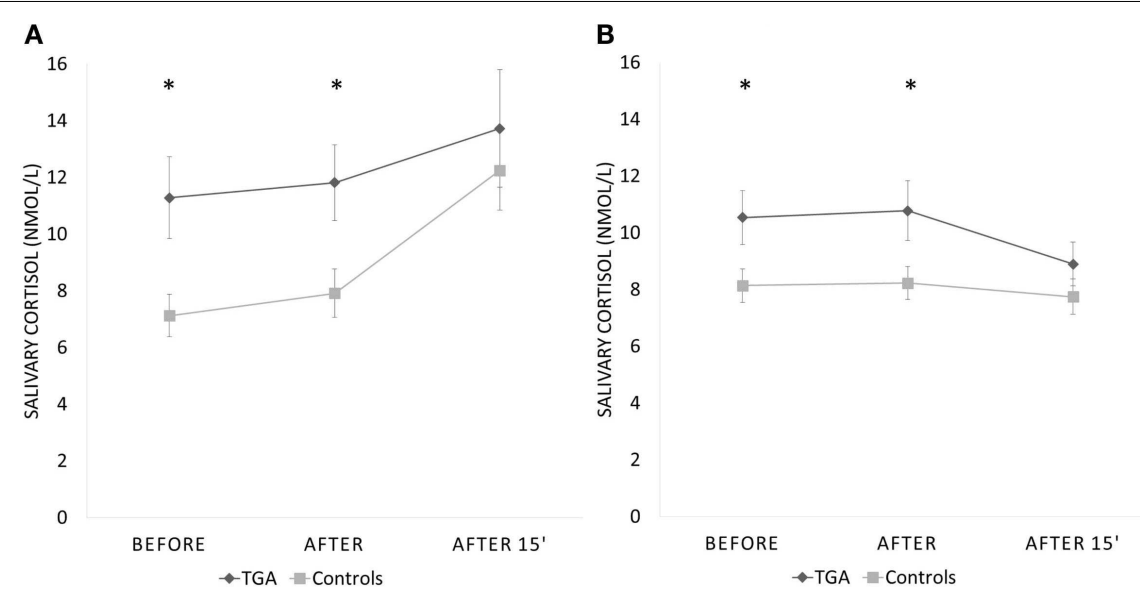

FIGURE 3 | Cortisol response to experimental stressor. Salivary cortisol response (in nmol/l) to the socially evaluated cold pressor test (A) and the control procedure with warm water (B) in 20 participants with a history of TGA and 20 normal controls. Data are presented as mean \pm standard error of the mean. ${ }^{*}$ Indicates significant group difference.

Table 3 | Personality trait evaluation

\begin{tabular}{lcrc}
\hline & TGA & Controls & $\boldsymbol{p}$ value \\
\hline Self-report scales & & & \\
CES-D, sum; mean (SEM) & $12.3( \pm 1.19)$ & $8.5( \pm 1.36)$ & $\mathbf{0 . 0 2 1}$ \\
STAI sum; mean (SEM) & $39.3( \pm 1.96)$ & $31.0( \pm 2.07)$ & $\mathbf{0 . 0 0 7}$ \\
TICS sum; mean (SEM) & $67.15( \pm 3.77)$ & $59.2( \pm 3.08)$ & 0.134 \\
\hline
\end{tabular}

CES-D, Center for Epidemiological Studies Depression Scale; SEM, standard error of the mean; STAI, state-trait anxiety scale; TICS, trier inventory for chronic stress. Bold font indicates $p<0.05$.

higher self-reported anxiety in our patients strengthens this possible mechanism. In contrast, the reactive cortisol response $15 \mathrm{~min}$ after the SECPT was not significantly different between the groups.

Recent research suggests that the interaction of genetic and cognitive or emotional factors in which glucocorticoid hormones and receptors play a crucial role might be responsible for the differences of coping with and adaptation to stress in individuals (Oitzl et al., 2010). In selected mental disorders, including major depressive disorder, posttraumatic stress disorder, and borderline personality disorder, the interaction of stress, the hippocampus, and memory function is well characterized by alterations of the HPA axis and hippocampal dysfunctions (Wingenfeld and Wolf, 2014). We observed an alteration of the HPA axis regulation with a stronger suppression after dexamethasone pre-treatment in our collective of TGA patients, a pattern consistently reported in patients with posttraumatic stress disorder, opposed to non-suppression in patients with major depressive disorder (Handwerger, 2009). Analogous to previous observations in posttraumatic stress disorder (Yehuda et al., 2004) we assume that the depressive status in the TGA group did not impact the HPA axis activity. Last but not least the intact cortisol awakening response is in sharp contrast to the absent cortisol awakening response occurring in patients with global (non-transient) amnesia (Wolf et al., 2005) and in patients with hippocampal damage (Buchanan et al., 2004).
Our findings in participants with a previous TGA indicate a hypersensitivity of the HPA axis with stronger pharmacological suppression and higher cortisol levels in anticipation of experimental stress. This neuroendocrine pattern shares similarities with stress and trauma associated psychiatric disorders. In TGA, the enhanced stress sensitivity may result in the pathophysiological activation of metabolic responses affecting the function of the hippocampus on a cellular level. Activation of the HPA axis during accidental or experimentally induced stress is known to lead to an elevation of glucocorticoid hormone levels. High glucocorticoid levels in turn have been shown to increase neuronal vulnerability in the hippocampus, to induce a decrease in regional cerebral blood flow in the mesial temporal lobe, and to have a negative effect on cognition and memory (Lupien and Lepage, 2001). Our findings support the possible role of a stress-induced cascade of steroid-mediated glutamatergic cytotoxicity, effecting the structural integrity of CA-1 neurons in the hippocampus (Bartsch and Deuschl, 2010).

For critical interpretation of our data, we assessed the possible effect of the identified personality traits of the TGA group on the cortisol values during experimental stress exposure but found no correlation between either the first cortisol value or the stress reactivity and the CES-D, STAI or TICS scores. Previous studies found no close relationship between personality factors and stressinduced salivary cortisol increases (Kudielka et al., 2009). However, we cannot differentiate whether our findings are a prerequisite condition for or possibly the consequence of a TGA episode.

In conclusion, our findings reveal a hypersensitivity of the HPA axis and suggest that an individual stress sensitivity might play a role in the pathophysiology of TGA.

\section{AUTHOR CONTRIBUTIONS}

MG, FN, and KS contributed to study conception and design, data collection and analysis, interpretation, drafting, and revising the manuscript. BG and AE contributed to data collection and analysis and revision of the manuscript. HF contributed to study 
conception and design, data analysis, interpretation, and revision of the manuscript. OW contributed to data analysis, interpretation, and revision of the manuscript. AG and $\mathrm{MH}$ contributed to study conception and design, data analysis, and revision of the manuscript. All authors have approved the final manuscript version to be published and have agreed to be accountable for all aspects of the work in ensuring that questions related to the accuracy or integrity of any part of the work are appropriately investigated and resolved.

\section{ACKNOWLEDGMENTS}

Funding for this study was provided by project $\mathrm{C} 07$ of the Deutsche Forschungsgemeinschaft (DFG) Collaborative Research Center 636 "Learning, memory and brain plasticity: implications for psychopathology."

\section{REFERENCES}

Alpers, G. W., Abelson, J. L., Wilhelm, F. H., and Roth, W. T. (2003). Salivary cortisol response during exposure treatment in driving phobics. Psychosom. Med. 65, 679-687. doi:10.1097/01.PSY.0000073872.85623.0C

Aschenbrenner, S., Tucha, O., and Lange, K. W. (2001). Regensburg Vocabulary Test/Regensburger Wortflüssigkeitstest. Goettingen: Hogrefe.

Baracchini, C., Tonello, S., Farina, F., Viaro, F., Atzori, M., Ballotta, E., et al. (2012). Jugular veins in transient global amnesia: innocent bystanders. Stroke 43, 2289-2292. doi:10.1161/STROKEAHA.112.654087

Bartsch, T., Alfke, K., Deuschl, G., and Jansen, O. (2007). Evolution of hippocampal CA-1 diffusion lesions in transient global amnesia. Ann. Neurol. 62, 475-480. doi:10.1002/ana.21189

Bartsch, T., Alfke, K., Stingele, R., Rohr, A., Freitag-Wolf, S., Jansen, O., et al. (2006). Selective affection of hippocampal CA-1 neurons in patients with transient global amnesia without long-term sequelae. Brain 129(Pt 11), 2874-2884. doi:10.1093/brain/awl248

Bartsch, T., and Deuschl, G. (2010). Transient global amnesia: functional anatomy and clinical implications. Lancet Neurol. 9, 205-214. doi:10.1016/S14744422(09)70344-8

Bremner, J. D., Vythilingam, M., Vermetten, E., Adil, J., Khan, S., Nazeer, A., et al. (2003). Cortisol response to a cognitive stress challenge in posttraumatic stress disorder (PTSD) related to childhood abuse. Psychoneuroendocrinology 28, 733-750. doi:10.1016/S0306-4530(02)00067-7

Buchanan, T. W., Kern, S., Allen, J. S., Tranel, D., and Kirschbaum, C. (2004). Circadian regulation of cortisol after hippocampal damage in humans. Biol. Psychiatry 56, 651-656. doi:10.1016/j.biopsych.2004.08.014

CANTABeclipse. (2006). Test Administration Guide. Cambridge, MA: Cambridge Cognition Limited.

Castellani, J. W., Young, A. J., Sawka, M. N., Backus, V. L., and Canete, J. J. (1998). Amnesia during cold water immersion: a case report. Wilderness Environ. Med. 9, 153-155. doi:10.1580/1080-6032(1998)009[0153:ADCWIA]2.3.CO;2

de Timary, P., Roy, E., Luminet, O., Fillee, C., and Mikolajczak, M. (2008). Relationship between alexithymia, alexithymia factors and salivary cortisol in men exposed to a social stress test. Psychoneuroendocrinology 33, 1160-1164. doi:10.1016/j.psyneuen.2008.06.005

Dohring, J., Schmuck, A., and Bartsch, T. (2014). Stress-related factors in the emergence of transient global amnesia with hippocampal lesions. Front. Behav. Neurosci. 8:287. doi:10.3389/fnbeh.2014.00287

Engert, V., Efanov, S. I., Duchesne, A., Vogel, S., Corbo, V., and Pruessner, J. C. (2013). Differentiating anticipatory from reactive cortisol responses to psychosocial stress. Psychoneuroendocrinology 38, 1328-1337. doi:10.1016/j.psyneuen.2012. 11.018

Faglioni, P., Scarpa, M., Colombo, A., Botti, C., and Grisanti, A. (1992). A modelbased study of learning and memory following transient global amnesia attacks. Cortex 28, 9-22. doi:10.1016/S0010-9452(13)80162-1

Fisher, C. M., and Adams, R. D. (1964). Transient global amnesia. Acta Neurol. Scand. Suppl. 40(Suppl. 9), 1-83.

Folstein, M. F., Folstein, S. E., and McHugh, P. R. (1975). "Mini-mental state". A practical method for grading the cognitive state of patients for the clinician. $J$. Psychiatr. Res. 12, 189-198. doi:10.1016/0022-3956(75)90026-6
Frederiks, J. A. (1993). Transient global amnesia. Clin. Neurol. Neurosurg. 95, 265-283. doi:10.1016/0303-8467(93)90102-M

Gass, A., Gaa, J., Hirsch, J., Schwartz, A., and Hennerici, M. G. (1999). Lack of evidence of acute ischemic tissue change in transient global amnesia on singleshot echo-planar diffusion-weighted MRI. Stroke 30, 2070-2072. doi:10.1161/ 01.STR.30.10.2070

Griebe, M., Bazner, H., Kablau, M., Hennerici, M. G., and Szabo, K. (2015). Transient global amnesia in legal proceedings. Int. J. Legal Med. 129(1), 223-6. doi:10.1007/s00414-014-1038-5

Handwerger, K. (2009). Differential patterns of HPA activity and reactivity in adult posttraumatic stress disorder and major depressive disorder. Harv. Rev. Psychiatry 17, 184-205. doi:10.1080/10673220902996775

Härting, C., Markowitsch, H. J., Neufeld, H., Calabrese, P., Deisinger, K., and Kessler, J. (2000). German Version of the Wechsler Memory Scale - Revised. Bern: Hans Huber.

Hautzinger, M., and Bailer, M. (1993). Allgemeine Depressions Skala - ADS. Weinheim: Beltz.

Helmstaedter, C. L. M., and Lux, S. (2001). VLMT (Verbaler Lern- und Merkfaehigkeitstest): Manual. Goettingen: Beltz Test GmbH.

Hodges, J. R., and Warlow, C. P. (1990). The aetiology of transient global amnesia. A case-control study of 114 cases with prospective follow-up. Brain 113(Pt 3), 639-657. doi:10.1093/brain/113.3.639

Horn, W. (1983). Achievement Measure System (Leistungs-Pruef-System/LPS). Goettingen: Hogrefe.

Inzitari, D., Pantoni, L., Lamassa, M., Pallanti, S., Pracucci, G., and Marini, P. (1997). Emotional arousal and phobia in transient global amnesia. Arch. Neurol. 54, 866-873. doi:10.1001/archneur.1997.00550190056015

Jager, T., Szabo, K., Griebe, M., Bazner, H., Moller, J., and Hennerici, M. G. (2009). Selective disruption of hippocampus-mediated recognition memory processes after episodes of transient global amnesia. Neuropsychologia 47, 70-76. doi:10.1016/j.neuropsychologia.2008.08.019

Kritchevsky, M., and Squire, L. R. (1989). Transient global amnesia: evidence for extensive, temporally graded retrograde amnesia. Neurology 39(2 Pt 1), 213-218. doi:10.1212/WNL.39.2.213

Kritchevsky, M., Squire, L. R., and Zouzounis, J. A. (1988). Transient global amnesia: characterization of anterograde and retrograde amnesia. Neurology 38, 213-219. doi:10.1212/WNL.38.2.213

Kudielka, B. M., Hellhammer, D. H., and Wust, S. (2009). Why do we respond so differently? Reviewing determinants of human salivary cortisol responses to challenge. Psychoneuroendocrinology 34, 2-18. doi:10.1016/j.psyneuen.2008.10.004

Laux, L., Glanzmann, P., Schaffner, P., and Spielberger, C. D. (1981). Das State-TraitAngstinventar. Weinheim: Beltz.

Lupien, S. J., and Lepage, M. (2001). Stress, memory, and the hippocampus: can't live with it, can't live without it. Behav. Brain Res. 127, 137-158. doi:10.1016/ S0166-4328(01)00361-8

Martin, E. A. (1970). Transient global amnesia. A report of eleven cases, including five of amnesia at the seaside. Ir. J. Med. Sci. 3, 331-335.

Mazzucchi, A., and Parma, M. (1990). "Neuropsychological testing of transient global amnesia during attack and during follow-up," in Transient Global Amnesia and Related Disorders, ed. H. J. Markowitsch (Toronto: Hogrefe \& Huber Publishers), 152-167.

Neri, M., Andermarcher, E., De Vreese, L. P., Rubichi, S., Sacchet, C., and Cipolli, C. (1995). Transient global amnesia: memory and metamemory. Aging 7, 423-429.

Oitzl, M. S., Champagne, D. L., van der Veen, R., and de Kloet, E. R. (2010). Brain development under stress: hypotheses of glucocorticoid actions revisited. Neurosci. Biobehav. Rev. 34, 853-866. doi:10.1016/j.neubiorev.2009.07.006

Pantoni, L., Bertini, E., Lamassa, M., Pracucci, G., and Inzitari, D. (2005). Clinical features, risk factors, and prognosis in transient global amnesia: a follow-up study. Eur. J. Neurol. 12, 350-356. doi:10.1111/j.1468-1331.2004.00982.x

Pruessner, J. C., Kirschbaum, C., Meinlschmid, G., and Hellhammer, D. H. (2003). Two formulas for computation of the area under the curve represent measures of total hormone concentration versus time-dependent change. Psychoneuroendocrinology 28, 916-931. doi:10.1016/S0306-4530(02)00108-7

Quinette, P., Guillery, B., Desgranges, B., de la Sayette, V., Viader, F., and Eustache, F. (2003). Working memory and executive functions in transient global amnesia. Brain 126(Pt 9), 1917-1934. doi:10.1093/brain/awg201

Quinette, P., Guillery-Girard, B., Dayan, J., de la Sayette, V., Marquis, S., Viader, F., et al. (2006). What does transient global amnesia really mean? Review 
of the literature and thorough study of 142 cases. Brain 129, 1640-1658. doi:10.1093/brain/awl105

Schellig, D., and Schächtele, B. (2009). Visual and Verbal Memory Test/Visueller und Verbaler Merkfähigkeitstest. Göttingen: Hogrefe.

Schmidtke, K., and Ehmsen, L. (1998). Transient global amnesia and migraine. A case control study. Eur. Neurol. 40, 9-14. doi:10.1159/000007948

Schmidt-Reinwald, A., Pruessner, J. C., Hellhammer, D. H., Federenko, I., Rohleder, N., Schurmeyer, T. H., et al. (1999). The cortisol response to awakening in relation to different challenge tests and a 12-hour cortisol rhythm. Life Sci. 64, 1653-1660. doi:10.1016/S0024-3205(99)00103-4

Schulz, P., and Schlotz, W. (1999). Trierer Inventar zur Erfassung von chronischem Streß (TICS): Skalenkonstruktion, teststatistische Überprüfung und Validierung der Skala Arbeitsüberlastung. Diagnostica 45, 8-19. doi:10.1026//00121924.45.1.8

Schwabe, L., Haddad, L., and Schachinger, H. (2008). HPA axis activation by a socially evaluated cold-pressor test. Psychoneuroendocrinology 33, 890-895. doi:10.1016/j.psyneuen.2008.03.001

Sedlaczek, O., Hirsch, J. G., Grips, E., Peters, C. N., Gass, A., Wohrle, J., et al. (2004). Detection of delayed focal MR changes in the lateral hippocampus in transient global amnesia. Neurology 62, 2165-2170. doi:10.1212/01.WNL.0000130504. 88404.C9

Uttner, I., Weber, S., Freund, W., Schmitz, B., Ramspott, M., and Huber, R. (2007). Transient global amnesia - full recovery without persistent cognitive impairment. Eur. Neurol. 58, 146-151. doi:10.1159/000104715

Wingenfeld, K., and Wolf, O. T. (2014). Stress, memory, and the hippocampus. Front. Neurol. Neurosci. 34:109-120. doi:10.1159/000356423

Wolf, O. T., Fujiwara, E., Luwinski, G., Kirschbaum, C., and Markowitsch, H. J. (2005). No morning cortisol response in patients with severe global amnesia. Psychoneuroendocrinology 30, 101-105. doi:10.1016/j.psyneuen.2004.05.001
Yehuda, R., Halligan, S. L., Golier, J. A., Grossman, R., and Bierer, L. M. (2004). Effects of trauma exposure on the cortisol response to dexamethasone administration in PTSD and major depressive disorder. Psychoneuroendocrinology 29, 389-404. doi:10.1016/S0306-4530(03)00052-0

Zimmermann, P., and Fimm, B. (2012). Test for Attentional Performance/Testbatterie zur Aufmerksamkeitspruefung (TAP), 2.3 Edn. Freiburg: Psytest.

Conflict of Interest Statement: Frauke Nees and Kristina Szabo have received funding for this study from the Deutsche Forschungsgemeinschaft (DFG); project C07 of the Collaborative Research Center 636 "Learning, memory and brain plasticity: implications for psychopathology.” Martin Griebe, Benjamin Gerber, Anne Ebert, Herta Flor, Oliver T. Wolf, Achim Gass, and Michael G. Hennerici declare that the research was conducted in the absence of any commercial or financial relationships that could be construed as a potential conflict of interest.

Received: 17 January 2015; accepted: 23 February 2015; published online: 09 March 2015.

Citation: Griebe M, Nees F, Gerber B, Ebert A, Flor H, Wolf OT, Gass A, Hennerici $M G$ and Szabo K (2015) Stronger pharmacological cortisol suppression and anticipatory cortisol stress response in transient global amnesia. Front. Behav. Neurosci. 9:63. doi: $10.3389 /$ fnbeh.2015.00063

This article was submitted to the journal Frontiers in Behavioral Neuroscience. Copyright $\odot 2015$ Griebe, Nees, Gerber, Ebert, Flor, Wolf, Gass, Hennerici and Szabo. This is an open-access article distributed under the terms of the Creative Commons Attribution License (CC BY). The use, distribution or reproduction in other forums is permitted, provided the original author(s) or licensor are credited and that the original publication in this journal is cited, in accordance with accepted academic practice. No use, distribution or reproduction is permitted which does not comply with these terms. 